\title{
Cold-Spotting: Linking Primary Care and Public Health to Create Communities of Solution
}

\author{
John M. Westfall, MD, MPH
}

By providing enhanced primary care and social services to patients with high utilization of expensive emergency and hospital care, there is evidence that their health can improve and their costs can be lowered. This type of "hot-spotting" improves the care of individual patients. It may be that these patients live in communities with disintegrated social determinants of health, little community support, and poor access to primary care. These "cold spots" in the community may be amenable to interventions targeted at linking primary care and public health at broader community and population levels. Building local communities of solution that address the individual and population may help decrease these cold spots, thereby eliminating the hot spots as well. (J Am Board Fam Med 2013;26:239-240.)

Using medical billing data from hospitals in Camden, New Jersey, Dr. Jeffrey Brenner identified a small group of very high-cost patients. ${ }^{1}$ These "super-utilizers" repeatedly accessed emergency rooms, hospitals, and doctors' offices with complex medical conditions compounded by difficult social situations, and they often lived within small geographic areas-a neighborhood, city block, or single building. By providing and coordinating the individual medical care and social services for these "hot spots," Brenner and his group were able to reduce the cost of their care by up to half.

Today, identifying individual patients with high utilization of health care is relatively easy given the vast improvements in health information technology, electronic health records, and all-payor data bases. However, is the super-utilizer the real problem? Do they have some medical defect or general disregard for their community? Are hot spots the problem? Or is the problem really "cold spots," communities in which the social determinants of health, support, and access to primary care have broken down?

From the Department of Family Medicine, University of Colorado School of Medicine, Aurora, CO.

Funding: none.

Conflict of interest: none declared.

Corresponding author: John M. Westfall, Department of Family Medicine, University of Colorado School of Medicine, 12631 E. 17th Ave, Mail Stop F496, Aurora, CO 80045 (E-mail: Jack.Westfall@ucdenver.edu).

\section{Cold Spots}

Perhaps we could look at our system of care and identify the cold spots in our communities: those places—city blocks, urban neighborhoods, isolated rural towns- that have high usage of and expensive medical care. What is happening, or not happening, in the community that requires more expensive acute medical care? Cold-spotting finds communities, down to the city block, that do not provide the essential opportunities for health: safe sidewalks, good air quality, social integration, grocery stores, education, employment, public health.

Cold spots are common in poor urban communities similar to Camden, but they can exist among the endless rows of little boxes made of ticky-tacky of our suburbs and the gated communities of the affluent. They lack the warmth of social support. Cold spots contrast to communities that have low utilization, often neighborhoods with well-lit streets and smooth sidewalks, safe cul-de-sacs, green space, walking trails, and a vibrant economy that provides individuals and families with education, employment, and purpose. Communities with low utilization often are packed with community organizations that engage individuals, where neighbors know and support each other and social isolation is rare.

Hot-spotting identifies individuals with high utilization of emergency and hospital care. They need frequent and expensive treatment. The cure for hot spots is to provide more intense, low-cost 
ambulatory care and social services. Hot-spotting provides individualized services to improve individual health. It is effective at decreasing hospitalizations by replacing acute illness care with comprehensive chronic disease management, community health workers, and improved home care and selfmanagement. Dr. Brenner's approach promises to help thousands of individuals in our communities and should be replicated throughout the nation. But it should be no surprise that the first hot spot Dr. Brenner identified was in the disintegrated heart of inner-city Camden, New Jersey.

Whether hot-spotting or cold-spotting, the methods of identification are the same: find high utilization of expensive medical care. Hot spots can exist within cold-spot communities, but the solutions to cold spots are very different.

\section{Communities of Solution}

Cold-spotting identifies problem places and provides an opportunity for engaged community building. The social determinants of bad health will not be improved in sustainable ways without programs aimed at the block, community, town, city, hospital catchment area, or health problem shed. It is not about an individual-it is about a system, a culture, and a community. The 1967 Folsom Report proposed a broad frame work for supporting comprehensive personal health care, community health, social engagement, and workforce development, all anchored within a local "community of solution." The community of solution concept arose from the recognition that complex political and administrative structures often hinder local problem solving by creating barriers to the identification of local needs and local solutions. Today's pressing need to defragment and improve the value of health care requires a setting that fosters improvements in the social determinants of health. A strong community of solution improves the community, which in turn produces good jobs, effective education, safe housing, public health, and, yes, improved access to primary care. The recent renewal of the community of solution concept may be the anchor for identifying and mitigating cold spots in our communities and can lead to improved individual and population health. ${ }^{2}$ The recent Insti- tute of Medicine report on integrating primary care and public health ${ }^{3}$ provides additional encouragement to actively bring together essential partners to build programs that promote population health. The current issue of the Fournal of the American Board of Family Medicine provides several early examples of communities that are working to address the cold spots in their states and communities and describe the benefits to the population and the individual. The answer is not just additional money and resources, but in building a community of solution that addresses the social determinants of health.

We know this works. Dr. Brenner's approach provides evidence that when patients are engaged and provided with education, training, and opportunity, their health improves and they cost less. How can we take this evidence and apply it broadly, not just to individuals, but to neighborhoods through communities of solution? Cold-spotting requires a shift in our thinking, a shift evident in the projects described in this issue of the fournal. The health care problems and overrun costs are not due to individual patients. Our problems are systemic and community-based. The problem sheds are not in individual homes or with individual patients-hot spots. The problem sheds are larger, wider cold spots. The cure requires a community approach, linking public health and primary care in explicit partnerships that address the needs of the individual and build an environment and community that supports healthy living. Together, when we build a community of solution, we eliminate cold spots, which will also help eliminate our hot spots.

\section{References}

1. Gawande A. The hot spotters: can we lower medical costs by giving the neediest patients better care? The New Yorker, January 24, 2011.

2. Lesko S, Griswold K, David SP, et al. Communities of solution: the Folsom Report revisited. Ann Fam Med 2012;10:250-60.

3. Institute of Medicine. Primary care and public health: exploring integration to improve population health. Washington, D.C.: the National Academies Press; 2012. 